Methods Data come from the British Regional Heart Study (BRHS) and the Health, Aging and Body Composition (HABC) Study. The BRHS included older men from 24 British towns aged $71-92$ years in $2010-12(n=2,147)$. The HABC Study, included 3,075 American men and women aged 71-80. In both studies, measures of oral health included tooth loss, periodontal disease, dry mouth, and self-rated oral health. Dietary data included dietary quality (Elderly Dietary Index in the BRHS, and Healthy Eating Score in the HABC Study) and intake (processed meat, calories from fat, protein and fruits and vegetables). Additionally in the BRHS, change in dietary quality was assessed over 10 years from 1998-2000 (age 60-79 years) to 2010-2012 (71-92 years).

Results In the BRHS, tooth loss, fair/poor self-rated oral health and accumulation of oral health problems were associated with poor dietary quality, after adjustment for age, social class, smoking, alcohol, history of cardiovascular disease (CVD) and diabetes, body mass index (BMI) and energy intake. Similar associations were observed for high intake of processed meat. Accumulation of oral health problems and self-rated oral health were associated with being in the top quartile of percentage of calories from saturated fat (fair/poor self-rated oral health, odds ratio $(\mathrm{OR})=1.34$, 95\% CI 1.021.77) after adjustment for confounders. In the HABC study, no significant associations were observed between poor oral health and dietary quality after full adjustment. In the fullyadjusted model (age, gender, race, education, smoking, alcohol, history of CVD and diabetes, BMI and energy intake), periodontal disease was associated with the top quartile of percentage of calories from saturated fat $(\mathrm{OR}=1.48$, 95\%CI 1.092.01). In the BRHS, persistent low dietary quality over 10 years (from age 60-79 to 71-92 years), was associated with higher risk of tooth loss and accumulation of oral health problems at 71-92 years.

Conclusion Older individuals with oral health problems had poorer diets and consumed fewer nutrient-rich foods. Moreover, persistent poor dietary quality in older ages was associated with oral health problems later in life, suggesting bidirectional associations between oral health and dietary intake in older age. Improved management of nutrition and oral health are both important aspects of the health of older populations.

\section{P15 CHILDRENS' FOOD AND BEVERAGE PORTION SIZES ON THE ISLAND OF IRELAND: A QUALITATIVE STUDY OF PARENTS' VIEWS AND PRACTICES}

${ }^{1} \mathrm{JM}$ Harrington*, ${ }^{2} \mathrm{Cl}$ McGowan, ${ }^{2} \mathrm{M}$ O'Reilly, ${ }^{3} \mathrm{~S}$ Moore, ${ }^{3} \mathrm{JV}$ Woodside. ${ }^{1}$ School of Public Health, University College Cork, Cork, Ireland; ${ }^{2}$ Human Health and Nutrition Direcotrate, Safefood, Cork, Ireland; ${ }^{3}$ Northern Ireland Centre of Excellence for Public Health, Queens University, Belfast, UK

\subsection{6/jech-2020-SSMabstracts.111}

Background Portion sizes of commercially available foods and beverages have increased since the $1980 \mathrm{~s}$, as have portion sizes consumed by children. Children's consumption of larger portion sizes is associated with higher energy intakes and weight status. Parents and guardians act as critical role models for children in the development of eating habits and often decide the amounts (portion sizes) their children are served. How parents portion foods and beverages, and how they decide the amounts to serve is an understudied domain.
Children's portion sizes, thus presents a clear, modifiable determinant of excess energy consumption in children and risk of weight gain.

Methods The primary aim of this qualitative study, was to understand parents' practices in portioning food and beverages for their children; their mechanisms for judging appropriate portion sizes and the factors influencing these judgements. A sample of 144 parents with at least one child aged 2-12 years who did not require a diet on medical grounds participated. Parents were recruited via purposive sampling of preschools and primary schools geographically located in either urban or rural areas of Northern Ireland and the Republic of Ireland, and classified as either higher or lower levels of disadvantage.

Results Parents reported that they do not consciously think about the portion size (quantity) that they give to their children but place greater focus on the types of food served. Generally, parents feel that the portion sizes that they give to their children are appropriate. This was reflected in the three main themes comprising of multiple sub-themes that were identified from the analysis: 1) Parental portioning practices parent serves and permitting child to self-serve; 2) Factors influencing parental portioning including parent motivations, knowledge, child-related factors, family influences, school influences, food retail and other settings, socio-cultural influences and portioning resources used; 3) Parental views on portion size guidance including receptivity to portion size guidance and usefulness of guidance.

Conclusion Understanding how parents portion food for their children, the drivers of these practices and the type of information parents are receptive to will help inform future interventions and information campaigns to help parents understand child portion sizes. From this research it is evident that parents main concern regarding the amount that they feed their children is that their child eats enough to be fed. This amount is something that parents learn through experience of feeding their children and is highly specific to the appetite of each individual child.

\section{\begin{tabular}{|l|l}
\hline P16 & VEGETARIAN DIETS AND RISKS OF TOTAL AND
\end{tabular} SITE-SPECIFIC FRACTURES: RESULTS FROM THE PROSPECTIVE EPIC-OXFORD STUDY}

${ }^{1}$ TYN Tong*, ${ }^{1} \mathrm{PN}$ Appleby, ${ }^{2} \mathrm{MEG}$ Armstrong, ${ }^{1} \mathrm{GK}$ Fensom, ${ }^{1} \mathrm{~A}$ Knuppel, ${ }^{1} \mathrm{~K}$ Papier, ${ }^{1}$ A Perez-Cornago, ${ }^{1}$ RC Travis, ${ }^{1} \mathrm{TJ}$ Key. ${ }^{1}$ Cancer Epidemiology Unit, Nuffield Department of Population Health, University of Oxford, Oxford, UK; ${ }^{2}$ Centre for Exercise, Nutrition and Health Sciences, School for Policy Studies, University of Bristol, Bristol, UK

\subsection{6/jech-2020-SSMabstracts. 112}

Background There is limited prospective evidence on possible differences in fracture risks between meat-eaters and vegetarians. We aimed to study this in a prospective cohort with a large proportion of non-meat eaters.

Methods In EPIC-Oxford, dietary information was collected at baseline (1993-2001) and subsequently around 2010. Participants were categorised into five diet groups at both time points (with 20,106 regular meat-eaters: $\geq 50 \mathrm{~g}$ of meat per day, 9,274 low meat-eaters: $<50 \mathrm{~g}$ of meat per day, 8,037 fish-eaters, 15,499 vegetarians and 1,982 vegans at baseline for analyses of total fractures). Outcomes were identified through record linkage until mid-2016. Using multivariable Cox regression adjusted for socio-demographic, lifestyle confounders and body mass index (BMI), we estimated the risks 
of total $(n=3,941)$ and site-specific fractures (arm, $n=566$; wrist, $n=889$; hip, $n=945$; leg, $n=366$; ankle, $n=520$; other main sites i.e. clavicle, rib and vertebra, $n=467$ ) by diet group over a mean of 17.6 years of follow-up.

Results Compared with regular meat-eaters, vegetarians had marginally higher risks of total fractures (hazard ratios 1.10; $95 \%$ confidence interval 1.00-1.20) and arm fractures (1.28; 1.01-1.63), while vegans had higher risks of total fractures (1.44; 1.21-1.72), arm fractures $(1.60,1.01-2.54)$ and leg fractures $(2.06 ; 1.22-3.47)$. For hip fractures, the risks were significantly higher in fish-eaters $(1.28 ; 1.03-1.59)$, vegetarians $(1.27 ; 1.05-1.55)$ and vegans $(2.35 ; 1.67-3.30)$ compared with regular meat-eaters. There were no significant differences in risks of wrist, ankle or other main site fractures by diet group. Overall, the significant associations appeared to be stronger before adjustment for BMI (e.g. 1.52; 1.27-1.81 in vegans for total fractures), and were slightly attenuated but remained significant with additional adjustment for dietary calcium and total protein.

Conclusion Overall, non-meat eaters, especially vegans, had higher risks of either total or some site-specific fractures, particularly hip fractures, than regular meat eaters. These differences may be partially related to lower BMI or lower dietary intakes of calcium and protein in the non-meat eaters. Further studies of non-European and contemporary populations are needed to determine the generality of these findings.

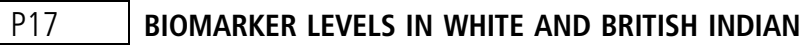 VEGETARIANS AND NON-VEGETARIANS IN THE UK BIOBANK}

${ }^{1}$ TYN Tong*, ${ }^{1}$ A Perez-Cornago, ${ }^{2}$ KE Bradbury, ${ }^{1}$ TJ Key. ${ }^{1}$ Cancer Epidemiology Unit, Nuffield Department of Population Health, University of Oxford, Oxford, UK; ${ }^{2}$ National Institute for Health Innovation, School of Population Health, Faculty of Medical and Health Sciences, University of Auckland, Auckland, New Zealand

\subsection{6/jech-2020-SSMabstracts.113}

Background A comprehensive description of disease biomarker levels in people of different habitual diet groups is lacking. We conducted cross-sectional analyses of mean biomarker concentrations by diet group in a large cohort.

Methods The UK Biobank recruited around 500,000 middle aged participants throughout the United Kingdom in 20062010. Blood and urine samples were collected from the majority of participants, and assayed for a range of serum and urinary biomarkers related to disease status of six outcomes (cardiovascular diseases, bone and joint health, cancer, diabetes, renal disease, and liver). Using multivariable linear regression adjusted for age, sex, fasting status, body mass index and lifestyle confounders, we estimated geometric mean biomarkers concentrations by six diet groups (221,295 regular meat-eaters, 222,038 low meat-eaters, 5,053 poultry eaters, 10,470 fish eaters, 6,804 vegetarians, 416 vegans) in white British participants, and two diet groups in British Indian participants (4091 meat eaters, 1444 vegetarians).

Results We observed differences in the concentrations of many biomarkers by diet group. The biomarkers with the largest percentage difference by extreme diet groups (i.e. vegans versus regular meat-eaters) within each disease outcome group in the white British population are reported below. Compared with white British regular meat-eaters, white British vegans had lower C-reactive protein $(1.10,1.00-1.21$ versus 1.43 , $1.43-1.44 \mathrm{mg} / \mathrm{L})$ and low density lipoprotein cholesterol (3.12, 3.06-3.19 versus $3.65,3.65-3.65 \mathrm{mmol} / \mathrm{L})$; lower vita$\min \mathrm{D}(34.3,33.0-35.8$ versus $44.5,44.4-44.5 \mathrm{nmol} / \mathrm{L})$; higher sex hormone-binding globulin $(51.1,48.9-53.3$ versus 45.0, 44.9-45.0 nmol/L); lower haemoglobin A1C (HbA1C, $33.8,33.4-34.2$ versus $35.2,35.2-35.2 \mathrm{mmol} / \mathrm{mol}$ ); lower urinary creatinine $(5389,5076-5723$ versus 7289, 7269-7308 $\mu \mathrm{mol} / \mathrm{L})$; and lower gamma glutamyltransferase (23.4, 22.124.7 versus 29.7, 29.6-29.8 U/L). Patterns were similar in British Indians, with the exception of HbA1C which was not significantly different between meat-eaters and vegetarians. In both ethnicities, the differences in biomarker levels by diet group were consistent between men and women.

Conclusion In this large population cohort, participants of different diet groups exhibited differences in many biomarkers. These biomarkers are associated with disease risk, and therefore the observed differences may be suggestive of differences in future disease risks by diet group, which should be further investigated.

\section{P18 STRUCTURAL ASPECTS OF SOCIAL CONNECTEDNESS AND ADHERENCE TO A HEART-HEALTHY DIET IN 60-64 YEAR OLDS IN THE NATIONAL SURVEY OF HEALTH AND DEVELOPMENT (NSHD)}

C Constable Fernandez*, J Maddock, P Patalay. MRC Lifelong Health and Ageing, University College London, London, UK

\subsection{6/jech-2020-SSMabstracts. 114}

Background Social connectedness has been shown to influence mortality to the same extent as other well-established risk factors such as smoking. Structural aspects of social connectedness include martial, cohabitation and employment status which are the focus of the present study. Dietary behaviour is a possible pathway through which social relationships exert their influence on health outcomes. Among those above 65 years, research has indicated that living alone and being socially isolated is a risk factor for poor diet variety. The association between retirement and diet quality is not well established, and at 60-64 years, the present study group represent an interesting time of transition in the life course. Moreover, evidence suggests social influences on dietary behaviour are sex-specific. This study aims to examine the association of structural aspects of social networks and diet quality in the 60-64 years age group.

Methods Participants came from the Medical Research Council National Survey of Health and Development (NSHD). The cohort has been followed up 24 times from birth, including a follow-up in 2006-10 at ages 60-64 years when 1,869 participants completed 5 -day prospective estimated diet diaries. Diet quality was assessed according to adherence of a Dietary Approaches to Stop Hypertension (DASH)-type diet score ranging from 0-40. Participants were asked, via questionnaire, to report their current marital status, the number of people living in their household and their current employment status.

Linear regression models were used to determine if marital status, the number of people living in the participant's household and employment status were associated with adherence to a DASH-type diet. Models were also stratified by sex. 